\title{
Sprunggelenksfraktur im Alter - was ist anders?
}

\author{
Sabine Ochman, Julia Evers, Michael J. Raschke
}

\section{Zusammenfassung}

Aufgrund der demografischen Entwicklung ist mit einer deutlichen $\mathrm{Zu}-$ nahme von Sprunggelenksfrakturen beim älteren Patienten zu rechnen. Im Vergleich zum jungen Patienten finden sich Unterschiede hinsichtlich des Traumamechanismus, des Frakturmusters, der Knochenqualität und der Nebenerkrankungen. Auch beim älteren Patienten steht die Wiederherstellung der Funktion an oberster Stelle des therapeutischen Regimes. Die konservative Therapie wird bei stabilen Frakturformen sowie instabilen Frakturen mit Kontraindikationen zur operativen Therapie empfohlen. Die operative Therapie sollte weichteiladaptiert und durch spezielle operative Techniken und Implantate individuell erfolgen. Hierbei hat die initiale Transfixation mittels Fixateur externe einen hohen Stellenwert. Die operativen Techniken reichen von dorsaler Plattenpositionierung über K-Draht-Techniken, Hakenplatten, Fibulanägel bis hin zu winkelstabilen Plattensystemen, die gute postoperative Ergebnisse zeigen. Eine primäre Versteifung mittels retrograder Nagelarthrodese ist nur als Salvage-Verfahren Ausnahmefällen vorbehalten.

\section{Ankle Fractures in the Elderly - What is Different?}

Because of the demographic changes there will be an increase of osteoporotic ankle fractures in the elderly. Differences to ankle fractues in younger patients are observed regarding the trauma mechanism, bone quality and comoribidities. The goal of operative treatment is reconstruction of the function and exact anatomy of the ankle joint with respect to the soft tissue trauma. Preoperatively CT scan is recommended for diagnosis and preoperative planning. Recommended operative techniques are the use of locking plates, dorsal plate positioning and second stage reconstruction with initial use of external fixation. Primary arthrodesis using a retrograde intramedullary nail should be considered as salvage procedure in exceptional cases.

\section{Einleitung}

Die Inzidenz von Frakturen des oberen Sprunggelenks bei älteren Patienten zeigt eine stetige Zunahme aufgrund der demografischen Entwicklung und es ist mit weiter steigenden Zahlen zu rechnen $[4,10]$. Vor diesem Hintergrund und der Zunahme der Aktivität im Alter mit An-

OP-JOURNAL 2014; 30: 76-80

(c) Georg Thieme Verlag KG Stuttgart · New York DOI http://dx.doi.org/10.1055/s-0034-1383260 spruch auf eine hohe Lebensqualität hat die adäquate Versorgung der Sprunggelenksfraktur des älteren Patienten eine zunehmende Bedeutung.

Die Sprunggelenksfraktur an sich ist jedoch keine typische osteoporotische Verletzung. Im Vordergrund bei Verletzungen des Sprunggelenks im Alter stehen neben der Knochenalterung altersbedingte Veränderungen der peripheren Nerven sowie der Gefäße, insbesondere zeigen sich die Auswirkungen der Neuropathie meist auf dem Boden endokri- ner Erkrankungen wie dem Diabetes mellitus. Aufgrund der Missempfindungen zeigen diese Patienten zusätzlich eine erhöhte Frakturgefährdung aufgrund der fehlenden Schmerzwahrnehmung als Schutzmechanismus [21]. Hinzu kommt die relative Immunschwäche durch den Diabetes mellitus mit der erhöhten Infektionsgefahr bei Weichteilbeteiligung und periphere Minderdurchblutung durch vaskuläre Komplikationen der Grunderkrankung. Unabhängig vom Diabetes zeigen viele ältere Patienten arterielle oder venöse Perfusionsstörungen. Im Rahmen der Verletzung der unteren Extremitäten haben diese insbesondere Einfluss auf das Ausmaß des Weichteilschadens und im Verlauf Einfluss auf die Wund- und Frakturheilung.

\section{Besonderheiten der Sprunggelenks- fraktur im Alter}

Im Vergleich zum jüngeren Patienten zeigen sich in nicht unerheblichem Maße Wundheilungsstörungen bis hin zur Nekrosenbildung und Gangrän. Durch venöse Abflussstörungen zeigen sich prolongierte Weichteilschwellungen, hinzu kommen vorbestehende trophische Störungen bis hin zu Ulzerationen, welche eine operative Versorgung und auch konservative Behandlung zunehmend erschweren [21].

Eine weitere Besonderheit im Alter besteht in bereits vorhandenen degenerativen Gelenkerkrankungen und Fehlstellungen. Diese können zum einen die Entstehung von Verletzungen durch Gangstörungen und verminderte $\mathrm{Ge}$ lenkmobilität begünstigen, zum anderen ist hierdurch das Risiko posttraumatischer Arthrosen erhöht bzw. die Rekonstruktion der vorgeschädigten Gelenkanatomie erschwert. Im weiteren Verlauf ist die Mobilisierung und Rehabilitation durch diese vorbestehenden Veränderungen beeinflusst und meist verlängert. 
Tab. 1 Altersspezifische Merkmale.

\begin{tabular}{ll} 
junger Patient & älterer Patient \\
\hline $\begin{array}{l}\text { Hochenergie- } \\
\text { trauma }\end{array}$ & $\begin{array}{l}\text { Niedrigenergie- } \\
\text { trauma }\end{array}$ \\
\hline $\begin{array}{l}\text { gute Knochen- } \\
\text { qualität }\end{array}$ & Osteoporose \\
\hline & $\begin{array}{l}\text { Defekt-, Mehrfrag- } \\
\text { mentfrakturen }\end{array}$ \\
\hline & $\begin{array}{l}\text { höhergradiger } \\
\text { Weichteilschaden }\end{array}$ \\
\hline & $\begin{array}{l}\text { trophische Störungen } \\
\text { Begleiterkrankungen }\end{array}$ \\
\hline & $\begin{array}{l}\text { vorbestehende } \\
\text { Fehlstellungen }\end{array}$ \\
\hline gute Compliance & $\begin{array}{l}\text { eingeschränkte } \\
\text { Compliance }\end{array}$
\end{tabular}

Im Gegensatz zu jüngeren Patienten zeigt sich als Traumamechanismus ein Niedrigenergietrauma, meist als Folge eines häuslichen Sturzes [24]. Weitere Besonderheiten sind aufgrund der osteoporotischen Knochenstruktur komplexe Frakturmuster mit mehrfragmentärem Charakter und Defekten sowie Trümmerzonen und Impressionen der Gelenkflächen [24]. Auffällig ist der hohe Anteil von PA-Frakturen (Pronation-Abduktion) im Stadium III nach LaugeHansen mit 90\%, wobei Pronationsfrakturen hoch signifikant als typische Altersfrakturen im mittleren Alter von 56,1 Jahren gegenüber SE (Supination-Eversion, 47,8 Jahre), PE (Pronation-Eversion, 47,7 Jahre) und SA (Supination-Abduktion, 41,3 Jahre) auftreten [35]. Vorbestehende trophische und vaskuläre Störungen sowie die dünne Hauttextur begünstigen fortgeschrittene Weichteilschäden und Weichteilkomplikationen im Verlauf (Tab. 1).

\section{Diagnostik}

Einen besonderen Stellenwert vor Therapieeinleitung hat die Eigen- oder Fremdanamnese bez. der Begleiterkrankungen wie Diabetes mellitus, peripherer arterieller Verschlusskrankheit, Osteoporose, neurologischen Defiziten, Dauermedikation (Antikoagulation, Kortikoide) und der Mobilitätsgrad vor dem Trauma [24].

Zur klinischen Untersuchung gehört die Überprüfung des neurovaskulären Status, inkl. einer Doppler-Untersuchung der peripheren Gefäße zur Erkennung von Perfusionsstörungen.
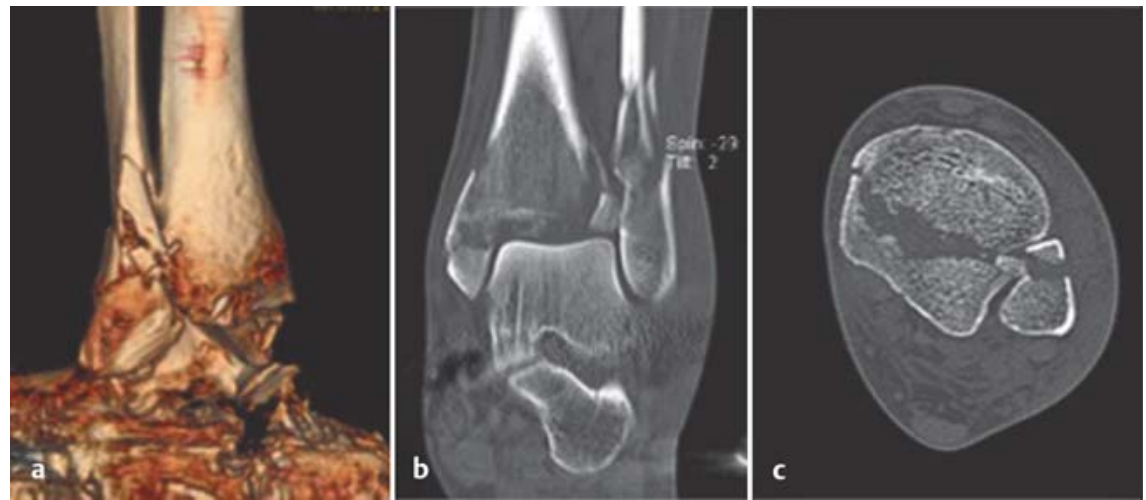

Abb. 1 a bis c Nachweis komplexerer Frakturmuster mittels computertomografischer Diagnostik bei osteoprotischer Knochenqualität mit Mehrfragmentfrakturen der Fibula (a, b), des Malleolus medialis (b) sowie Nachweis intermediärer Fragmente (b, c).

Besonderes Augenmerk sollte auf die Weichteilsituation gelegt werden, da diese entscheidend die Therapie und den Verlauf beeinflussen.

Die radiologische Diagnostik umfasst auch bei älteren Patienten die Standardprojektionen wie Mortise View und Aufnahme im lateralen Strahlengang.

Aufgrund der häufig zu verzeichnenden komplexen Frakturmuster ist eine ergänzende CT-Diagnostik anzuraten.

Nur mittels CT können Trümmer- und Defektzonen erkannt und die operative Therapie entsprechend geplant werden. Des Weiteren können knöcherne Ausrisse der Syndesmose sowie das Vorhandensein und das Ausmaß eines hinteren Kantenfragments häufig nur mittels CT diagnostiziert werden (Abb. 1).

Aufgrund der dünnen Weichteilummantelung des distalen Unterschenkels und der genannten trophischen Störungen im Alter ist eine sofortige Reposition bei Subluxation oder Dislokation erforderlich.

Anschließend erfolgt eine Retention, entweder in einer Schiene mit guter Polsterung oder durch eine Transfixation, insbesondere bei hochgradig instabilen Verletzungen, die sich im Gips nicht adäquat retinieren lassen, zur Stabilisierung und Weichteilkonsolidierung $[2,3,5]$. Zur Reduktion der Schwellung und Weichteilkonsolidierung sind abschwellende Maßnahmen dringend erforderlich. Zu nennen sind hier die konsequente Hochlagerung, Kühlung und insbesondere die Anwendung der intermittierenden Impulskompression.

\section{Therapieverfahren}

Im Gegensatz zum jüngeren Patienten steht eine frühe Mobilisierung des älteren Patienten im Vordergrund, der eine Teilbelastung i.d. R. nicht gewährleisten kann.

Die potenziellen Vorteile und Risiken einer konservativen Therapie müssen hier gegen das Risiko und die Komplikationen einer operativen Versorgung kalkuliert werden. Aufgrund der hohen Komplikationsraten nach operativer Therapie bestand der Trend zur konservativen Therapie der Sprunggelenksfraktur im Alter. Die Ergebnisse der konservativen Therapie hochgradig instabiler Frakturen zeigen jedoch Fehlverheilungen und Pseudarthrosen zwischen 48 und $73 \%[2,3]$. Studien, die operative und konservative Therapieverfahren miteinander verglichen, konnten nachweisen, dass die operative Therapie der Sprunggelenksfrakturen der konservativen überlegen war, da die eingebrachten Implantate die Frakturreposition halten konnten, bis die Konsolidierung eingetreten war $[3,14,32]$.

Die Komplikationsrate der operativ behandelten Patienten war höher; die konservativ behandelten Patienten hatten jedoch eine signifikant höhere Mortalität [14]. Allerdings konnte eine prospektiv-randomisierte Studie von Salai et al. zeigen, dass bei Patienten älter als 65 Jahre mit dislozierten Sprunggelenksfrakturen der AOFAS-Score bei den konservativ behandelten Patienten signifikant höher lag als bei den operativ behandelten [28]. Zusammen mit einer erhöhten Rehospitalisierungsrate bei den operativ behandelten Patienten [14] kann dies zu erhöhten Kosten führen [28]. 
Die operative Therapie der Sprunggelenksfraktur im Alter zeigt Komplikationsraten bis zu 21,5\% mit bis zu 10,8\% erforderlicher Majoramputation [22,33]. Die Rate an postoperativen Wundheilungsstörungen reicht von 7 bis $13 \%$ [16]. Neben den Wundheilungsstörungen kommt es in 5\% zu Fehlverheilungen und in $1 \%$ zu tiefen Infektionen [31] (Abb. 2).

Die Komplikationsraten steigen bei Vorhandensein von Komorbiditäten [16,23, 30].

Ermittelte Risikofaktoren für postoperative Komplikationen sind Nikotinkonsum, Alter, Diabetes mellitus, lokale Faktoren wie Osteoporose, periphere Neuropathie, periphere arterielle Verschlusskrankheit, Lymph- oder venöse Abflussstörung [33].

Die funktionellen Ergebnisse nach operativer Therapie erweisen sich jedoch als signifikant besser, sodass auch beim älteren Patienten die Kriterien zur operativen Therapiewahl gelten sollten [17, 31,35]. Stabile Frakturen sowie instabile Frakturen mit relativen Kontraindikationen bei schweren Komorbiditäten sowie reduziertem Allgemeinzustand können konservativ behandelt werden $[8,12,23$, 24,27,35]. Instabile Frakturen werden i.d. R. operativ behandelt.

Die Indikation beim älteren Patienten hängt jedoch, unabhängig vom radiologischen Befund, vom Weichteilbefund $\mathrm{ab}$.

Aufgrund der Begleiterkrankungen und dementsprechend verminderter Hauttextur, Perfusionsminderung sowie trophischer Störungen zeigen sich im Vergleich zu jüngeren Patienten häufiger zweit- bis drittgradig geschlossene Frakturen bzw. auch offene Frakturen. Eine operative Versorgung ist meist nicht innerhalb der erforderlichen 6-StundenGrenze möglich. Die Versorgung erfolgte erst verzögert aufgrund des ausgeprägten Weichteiltraumas.

\section{Operative Therapie}

Das primäre Ziel der operativen Therapie von Sprunggelenksfrakturen beinhaltet auch beim älteren Patienten eine präzise Wiederherstellung der talokruralen Anatomie und Gelenkflächen, Längenund Rotationswiederherstellung der Fibula sowie korrekte Positionierung der Fibula in die Inzisur der Tibia mit stabiler Fixierung.

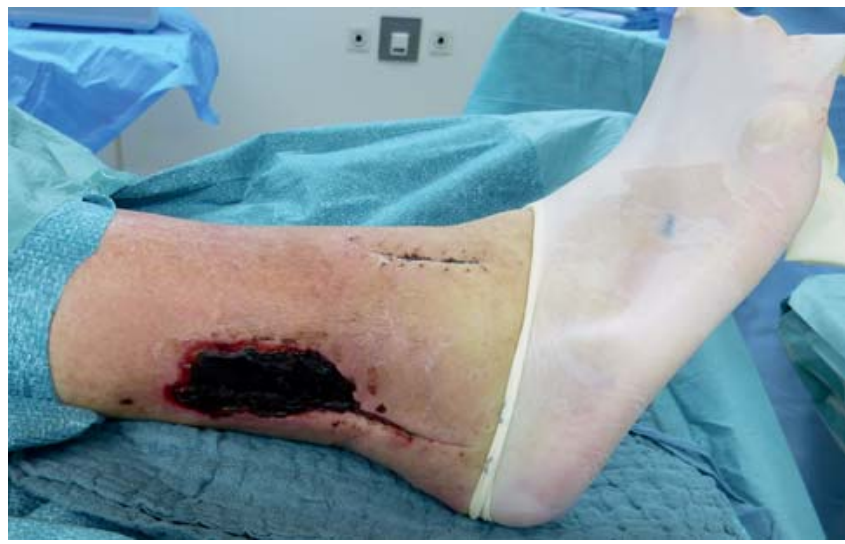

Abb. 2 Postoperative Nekrosenbildung im Bereich des lateralen Zugangs nach Plattenosteosynthese der Fibula (77-jährige Patientin, Marcumarisierung, Osteoporose).

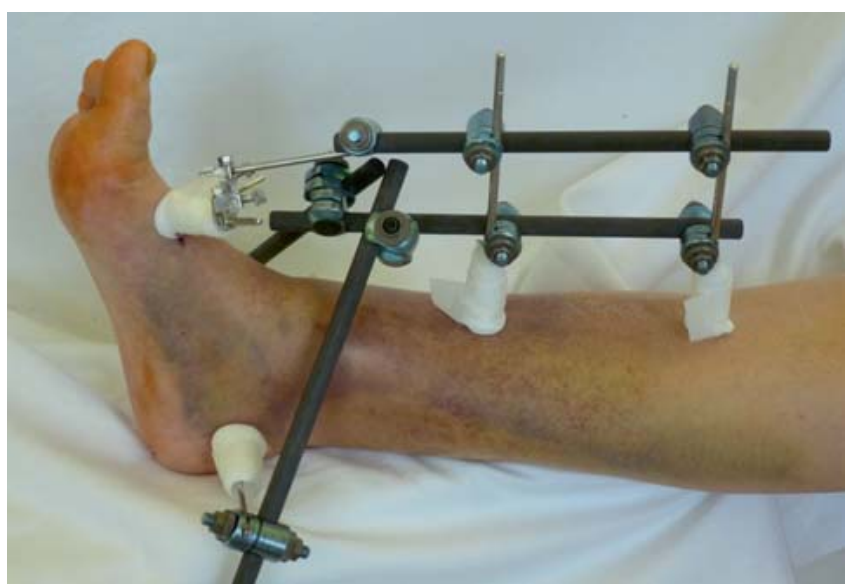

Abb. 3 Initiale Transfixation zur Konsolidierung der Weichteile und Stabilisierung (68-jährige Patientin).

Prinzipiell muss beim geriatrischen $\mathrm{Pa}$ tienten auf eine atraumatische Operationstechnik aufgrund der Weichteilund Hautkompromittierung geachtet werden [21].

In Abhängigkeit vom Weichteilbefund empfiehlt sich häufig ein zweizeitiges Vorgehen bei instabilen Frakturen durch initiale Transfixation und definitive Osteosynthese nach Weichteilkonsolidierung. (Abb.3). Eine Alternative bietet die temporäre Kirschner-Draht-Transfixation von plantar über den Kalkaneus und Talus in die distale Tibia [19].

Zur Protektion der posterioren Gelenkfläche des Subtalargelenks empfehlen Zwipp et al. dazu eine nicht parallele Anordnung der Kirschner-Drähte [35]. Die definitive osteosynthetische Versorgung stellt bei osteoporotischer Knochenqualität eine Herausforderung dar.

Lösungsansätze bieten eine posterolaterale Plattenpositionierung sowie winkelstabile Implantate im Bereich der Fibula $[7,11,27,35]$.

$\mathrm{Zu}$ bedenken bei der Implantatwahl ist weiterhin das Plattenprofil. Dieses sollte nicht zu kräftig sein, da der Haut-Weichteil-Mantel älterer Patienten ein Auftragen der Platten nicht problemlos toleriert und Folgen wie Nekrosen bis hin zu freiliegenden Implantaten auftreten können. In der aktuellen Literatur finden sich verschiedenste Lösungsansätze zur Optimierung der Versorgung osteoporotischer Sprunggelenksfrakturen. Eine Option bietet die dorsale Antigleitplatte mit biomechanischen Vorteilen bei osteoporotischer Knochenqualität sowie Vorteilen aufgrund der besseren Weichteildeckung $[20,29]$. Die Positionierung der Platte dorsal vermeidet prominente Schraubenköpfe lateral, die den ohnehin sehr dünnen Weichteilmantel kompromittieren können. Nachteilig kann die Irritation der Peronäalsehnen sein mit Erfordernis der sekundären Implantatentfernung [18].

Andere Arbeitsgruppen empfehlen die zusätzliche Fixierung der Fibula mit 2 parallelen 1,6-mm-Kirscher-Drähten im Kombination mit der konventionellen Drittelrohrplatte als sog. „K-wiretechnique“, welche biomechanisch eine signifikant höhere Versagenslast und Torsionsstabilität zeigte [13]. Das Einbringen mehrerer transfixierender fibu- 
lotibialer Schrauben als Tibia-pro-Fibula-Schraubenfixation zur Erhöhung der Stabilität ist eine weitere Alternative. Andere Arbeitsgruppen berichten über gute Erfahrungen mit der Hakenplatte [9,23], die Implantation eines Fibulaverriegelungsnagels [26] oder zusätzliche Augmentation mittels Kalziumphosphats [23]. Vorteile der Fibulanägel bestehen in der Option minimalinvasiver Zugänge, geringerer Weichteildissektion und geringerer Kompromittierung der Biologie der Frakturheilung [18]. Die Ergebnisse von wenigen Nachbeobachtungsstudien zeigen geringe Komplikationsraten bei guter Funktion und Patientenzufriedenheit $[6,25]$.

Einige Autoren propagieren eine Kombination aus 2 Platten, eine dorsal und eine lateral, zur Erhöhung der Stabilität bei Mehrfragmentfrakturen sowie längere Platten zur Verteilung des Stress Loads über eine längere Strecke [18]. Durch die zunehmende Verbreitung winkelstabiler Verfahren sind nun verschiedenste anatomisch angepasste, winkelstabile Low-Profile-Platten verfügbar, die insbesondere im distalen Anteil der Fibula eine winkelstabile Verankerung mehrerer Schrauben kleineren Durchmessers multidirektional erlauben (Abb. 4).

Biomechanische Untersuchungen konnten eine analoge Stabilität weniger winkelstabil unikortikal verankerter Schrauben im Vergleich zu mehreren bikortikal verankerten konventionellen Schrauben demonstrieren [11]. Die Stabilität einer winkelstabilen Frakturversorgung zeigt sich biomechanisch unabhängig von der Knochendichte (BMD) im Vergleich zur konventionellen Plattenosteosynthese [34].

Die Frakturen des medialen Malleolus zeigen beim älteren Patienten meist einen mehrfragmentären Charakter, sodass eine konventionelle Schraubenosteosynthese entweder technisch nicht möglich ist oder im Verlauf versagt. Hier bietet die Zuggurtungsosteosynthese oder eine Hakenplatte eine Alternative [18]. Bei degenerativen Vorschäden des Gelenks und erheblicher Gelenkflächenbeteiligung kann als Ultima Ratio eine primäre Arthrodese des Sprunggelenks durch einen retrograd implantierbaren Arthrodesennagel erfolgen $[1,15]$. Dieses gewährleistet eine stabile Frakturversorgung mit sofortiger Belastungsfähigkeit, ist jedoch nur als Salvage-Verfahren z. B. bei Patienten mit Osteoarthropathie oder zuvor bestehender Immobilität zu

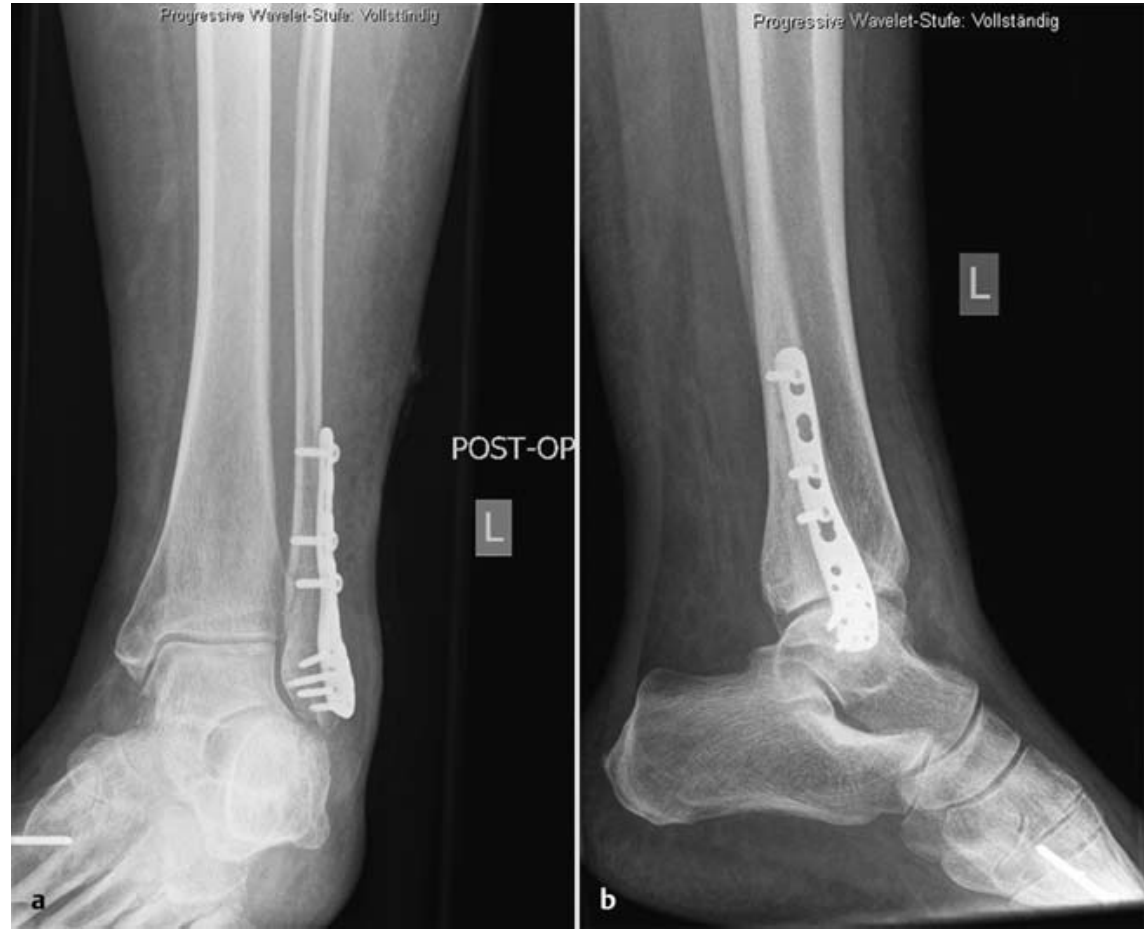

Abb.4a und b Lösungsansatz durch Einsatz winkelstabiler Implantate bei osteoporotischer Knochenstruktur.

sehen, da das nicht betroffene Subtalargelenk mitversteift wird und so erhebliche Funktionseinschränkungen im Kauf genommen werden [27].

\section{Nachbehandlung}

Die Nachbehandlung nach operativer Stabilisierung von Sprunggelenksfrakturen unterscheidet sich generell nicht von der jüngerer Patienten, ist jedoch weichteiladaptiert und in Abhängigkeit von der intraoperativen Knochenqualität zu steuern.

Direkt postoperativ ist auf eine ausreichende Hochlagerung und abschwellende Maßnahmen zu achten. Eine passivaktive Mobilisierung des Sprunggelenks sollte bereits frühzeitig erfolgen. In den ersten 6 Wochen erfolgt eine Mobilisation unter Teilbelastung für $20 \mathrm{~kg}$ im Gips/ Walker, Vollbelastung kann ab der 6 . postoperativen Woche erreicht werden. Häufig ist eine Teilbelastung der älteren Patienten nicht möglich. Dementsprechend empfiehlt sich zur externen Protektion neben der internen Fixierung eine Nachbehandlung im Gips oder Walker. Im postoperativen Verlauf ist, wie prä- und perioperativ, besonderes Augenmerk auf die Weichteilsituation zu legen. Verbände sollten nicht zu eng angelegt, auf Pflasterverbände mit Klebestreifen sollte verzichtet und die Extre- mität sollte gut gepolstert gelagert werden. Aufgrund der meist vorbestehenden vaskulären Störungen ist auf eine ausreichende Thromboembolieprophylaxe inkl. der intermittierenden Impulskompression zu achten.

Bei initialer tibiotarsaler Transfixation empfiehlt sich, diese nach osteosynthetischer Versorgung auch postoperativ zu belassen, zum einen zur Konsolidierung der Weichteile, zum anderen bei Patienten mit Demenz oder postoperativen Verwirrtheitszuständen [35]. Die weitere Nachbehandlung bei dementen Patienten sollte dann nach Fixateurentfernung im Gips für 4-6 Wochen erfolgen.

Bei osteoporotischer Knochenqualität und inadäquatem Traumamechanismus sollte eine Therapie gemäß der Leitlinie der DVO zur Frakturprophylaxe mit Kalzium und Vitamin $\mathrm{D}_{3}$ und nach entsprechender Basisdiagnostik durch Osteoporosetherapeutika wie Bisphosphonate nach Abschluss der Frakturheilung erfolgen.

\section{Fazit}

Aufgrund der demografischen Entwicklung ist bereits eine Zunahme der Sprunggelenksfrakturen beim älteren Patienten zu verzeichnen und es ist mit einer Verdreifachung bis zum Jahr 2030 
zu rechnen [10]. Um dem Rechnung zu tragen, müssen verschiedene Aspekte bei der Wahl des Therapieregimes beachtet werden. Die Behandlung dieser Verletzungen stellt aufgrund der oftmals kompromittierten Weichteilverhältnisse, der schlechten Knochenqualität, vorbestehender Deformitäten sowie degenerativer Veränderungen, der Notwendigkeit einer Frühmobilisation und Vermeidung einer längeren Immobilisation eine Herausforderung dar. Neben den Komorbiditäten des Patienten, das soziale Umfeld und die zuvor bestehende Mobilität hat die osteoporotische Knochenqualität Einfluss auf die Therapiewahl und die operativen Techniken und Implantate. Aus sozialökonomischer Sicht kann eine Verletzung des Sprunggelenks oder Fußes gravierende Folgen haben mit Verlust der prätraumatischen Selbstständigkeit, Funktionalität und Mobilität. Infolgedessen steht die Wiederherstellung der Funktion an oberster Stelle des therapeutischen Regimes. Die konservative Therapie wird bei stabilen Frakturformen sowie instabilen Frakturen mit Kontraindikationen zur operativen Therapie empfohlen. Die operative Therapie sollte weichteiladaptiert und durch spezielle operative Techniken und Implantate individuell erfolgen. Hierbei hat die initiale Transfixation einen hohen Stellenwert, die operativen Techniken reichen von dorsaler Plattenpositionierung über K-Wire-Techniken, Hakenplatten, Fibulanägel bis hin zu winkelstabilen Plattensystemen, die gute postoperative Ergebnisse zeigen. Eine primäre retrograde Nagelarthrodese ist nur als Salvage-Verfahren Ausnahmefällen vorbehalten.

\section{Literatur}

${ }^{1}$ Al-Nammari SS, Dawson-Bowling S, Amin A et $a l$. Fragility fractures of the ankle in the frail elderly patient: treatment with a long calcaneotalotibial nail. Bone Joint J 2014; 96-B: 817-822

2 Ali MS, McLaren CA, Rouholamin E et al. Ankle fractures in the elderly: nonoperative or operative treatment. J Orthop Trauma 1987; 1 : 275-280

${ }^{3}$ Anand N, Klenerman L. Ankle fractures in the elderly: MUA versus ORIF. Injury 1993; 24: 116-120

${ }^{4}$ Bauer M, Bengner U, Johnell O et al. Supination-eversion fractures of the ankle joint: changes in incidence over 30 years. Foot Ankle 1987; 8: 26-28
${ }^{5}$ Buckingham R, Hepple S, Winson I. Outcome of ankle fractures in the elderly. Foot and Ankle Surgery 2000; 6: 175-178

${ }^{6}$ Bugler KE, Watson CD, Hardie AR et al. The treatment of unstable fractures of the ankle using the Acumed fibular nail: development of a technique. J Bone Joint Surg Br 2012; 94: 1107-1112

${ }^{7}$ Cole PA, Craft JA. Treatment of osteoporotic ankle fractures in the elderly: surgical strategies. Orthopedics 2002; 25: 427-430

8 Giannini S, Chiarello E, Persiani V et al. Ankle fractures in elderly patients. Aging Clin Exp Res 2013; 25 (Suppl. 1): S77-S79

${ }^{9}$ Heim D, Niederhauser K. One-third tubular hook plate. Oper Orthop Traumatol 2007; 19: 305-309

${ }^{10}$ Kannus P, Palvanen M, Niemi S et al. Increasing number and incidence of low-trauma ankle fractures in elderly people: Finnish statistics during 1970-2000 and projections for the future. Bone 2002; 31: 430-433

${ }^{11} \mathrm{Kim}$ T, Ayturk UM, Haskell A et al. Fixation of osteoporotic distal fibula fractures: a biomechanical comparison of locking versus conventional plates. J Foot Ankle Surg 2007; 46: 2-6

12 Koval KJ, Lurie J, Zhou W et al. Ankle fractures in the elderly: what you get depends on where you live and who you see. J Orthop Trauma 2005; 19: 635-639

${ }^{13}$ Koval KJ, Petraco DM, Kummer FJ et al. A new technique for complex fibula fracture fixation in the elderly: a clinical and biomechanical evaluation. J Orthop Trauma 1997; 11: 28-33

14 Koval KJ, Zhou W, Sparks MJ et al. Complications after ankle fracture in elderly patients. Foot Ankle Int 2007; 28: 1249-1255

15 Lemon M, Somayaji HS, Khaleel A et al. Fragility fractures of the ankle: stabilisation with an expandable calcaneotalotibial nail. J Bone Joint Surg Br 2005; 87: 809-813

16 Lynde MJ, Sautter T, Hamilton GA et al. Complications after open reduction and internal fixation of ankle fractures in the elderly. Foot Ankle Surg 2012; 18: 103-107

${ }^{17}$ Makwana NK, Bhowal B, Harper WM et al. Conservative versus operative treatment for displaced ankle fractures in patients over 55 years of age. A prospective, randomised study. J Bone Joint Surg Br 2001; 83: 525529

18 McKean J, Cuellar DO, Hak D et al. Osteoporotic ankle fractures: an approach to operative management. Orthopedics 2013; 36: 936940

${ }^{19}$ Meier C, Schefold JC, Hug U. Temporary Kirschner wire ankle transfixation and delay ORIF. A staged operative treatment for closed unsatble ankle fractures with tibio-taler dislocations snd soft tissue trauma. Eur J Trauma 2004; 30: 371-377

20 Minihane KP, Lee C, Ahn C et al. Comparison of lateral locking plate and antiglide plate for fixation of distal fibular fractures in osteoporotic bone: a biomechanical study. J Orthop Trauma 2006; 20: 562-566

${ }^{21}$ Ochman S, Raschke MJ. Sprunggelenk und Fuß. Alterstraumatologie 2009; 1: 497-517

22 Pagliaro AJ, Michelson JD, Mizel MS. Results of operative fixation of unstable ankle fractures in geriatric patients. Foot Ankle Int 2001; 22: 399-402
${ }^{23}$ Panchbhavi VK, Mody MG, Mason WT. Combination of hook plate and tibial pro-fibular screw fixation of osteoporotic fractures: a clinical evaluation of operative strategy. Foot Ankle Int 2005; 26: 510-515

${ }^{24}$ Pichl J, Hoffmann R. [Ankle fractures in the elderly]. Unfallchirurg 2011; 114: 681-687

${ }^{25}$ Rajeev A, Senevirathna S, Radha S et al. Functional outcomes after fibula locking nail for fragility fractures of the ankle. Foot and Ankle Surgery 2011; 50: 547-550

${ }^{26}$ Ramasamy PR, Sherry $P$. The role of a fibular nail in the management of Weber type B ankle fractures in elderly patients with osteoporotic bone-a preliminary report. Injury 2001; 32: 477-485

${ }^{27}$ Rammelt S, Heim D, Hofbauer LC et al. [Problems and controversies in the treatment of ankle fractures]. Unfallchirurg 2011; 114: 847-860

${ }^{28}$ Salai M, Dudkiewicz I, Novikov I et al. The epidemic of ankle fractures in the elderly-is surgical treatment warranted? Arch Orthop Trauma Surg 2000; 120: 511-513

${ }^{29}$ Schaffer JJ, Manoli A, 2nd. The antiglide plate for distal fibular fixation. A biomechanical comparison with fixation with a lateral plate. J Bone Joint Surg Am 1987; 69: 596-604

30 Shivarathre DG, Chandran P, Platt SR. Operative fixation of unstable ankle fractures in patients aged over 80 years. Foot Ankle Int 2011; 32: 599-602

${ }^{31}$ Srinivasan CM, Moran CG. Internal fixation of ankle fractures in the very elderly. Injury 2001; 32: 559-563

32 Wronka KS, Salama H, Ramesh B. Management of displaced ankle fractures in elderly patients-is it worth performing osteosynthesis of osteoporotic bone? Orthop Traumatol Rehabil 2011; 13: 293-298

33 Zaghloul A, Haddad B, Barksfield R et al. Early complications of surgery in operative treatment of ankle fractures in those over 60: a review of 186 cases. Injury 2014; 45: 780-783

${ }^{34}$ Zahn RK, Frey S, Jakubietz RG et al. A contoured locking plate for distal fibular fractures in osteoporotic bone: a biomechanical cadaver study. Injury 2012; 43: 718-725

35 Zwipp H, Amlang M. [Treatment of fractures of the ankle in the elderly]. Orthopade 2014; 43: $332-338$

Priv.-Doz. Dr. Sabine Ochman Geschäftsführende Oberärztin Dr. med. Julia Evers

Assistenzärztin

Prof. Dr. med. Michael J. Raschke Klinikdirektor

Klinik für Unfall-, Hand- und Wiederherstellungschirurgie Universitätsklinikum Münster Albert-Schweitzer-Campus, W1 48149 Münster

Tel.: 0251/8356301

Fax: 0251/8356318

ochman@uni-muenster.de 